\title{
Everybody Nose: Molecular and Clinical Characteristics of Nasal Colonization During Active Methicillin-Resistant Staphylococcus Aureus Bloodstream Infection
}

\section{Erika Reategui Schwarz}

Icahn School of Medicine at Mount Sinai

Adriana van de Guchte

Icahn School of Medicine at Mount Sinai

Amy C. Dupper

Icahn School of Medicine at Mount Sinai

Ana Berbel Caban

Icahn School of Medicine at Mount Sinai

Devika Nadkarni

Icahn School of Medicine at Mount Sinai

Lindsey Fox

Icahn School of Medicine at Mount Sinai

Alexandra Mills

Icahn School of Medicine at Mount Sinai

Ajay Obla

Icahn School of Medicine at Mount Sinai

Kieran I. Chacko

Icahn School of Medicine at Mount Sinai

Irina Oussenko

Icahn School of Medicine at Mount Sinai

Flora Samaroo

Icahn School of Medicine at Mount Sinai

Jose Polanco

Icahn School of Medicine at Mount Sinai

Richard Silvera

Icahn School of Medicine at Mount Sinai

Melissa Smith

Icahn School of Medicine at Mount Sinai

Gopi Patel

Icahn School of Medicine at Mount Sinai 


\section{Melissa Gitman}

Icahn School of Medicine at Mount Sinai

Marilyn Chung

Icahn School of Medicine at Mount Sinai

Mitchell J. Sullivan

Icahn School of Medicine at Mount Sinai

Harm van Bakel

Icahn School of Medicine at Mount Sinai

Deena R. Altman ( $\boldsymbol{D}$ deena.altman@mssm.edu )

Icahn School of Medicine at Mount Sinai

\section{Research Article}

Keywords: methicillin-resistant Staphylococcus aureus, bloodstream infections, colonization, molecular epidemiology

Posted Date: December 2nd, 2021

DOI: https://doi.org/10.21203/rs.3.rs-1112075/v1

License: (c) (i) This work is licensed under a Creative Commons Attribution 4.0 International License. Read Full License 


\section{Abstract}

Background. Healthcare-associated infections pose a potentially fatal threat to patients worldwide and Staphylococcus aureus is one of the most common causes of healthcare-associated infections. S. aureus is a common commensal pathogen and a frequent cause of bacteremia, with studies demonstrating that nasal and blood isolates from single patients match more than $80 \%$ of the time. Here we report on a contemporary collection of colonizing isolates from those with methicillin-resistant $S$. aureus (MRSA) bloodstream infections to evaluate the diversity within hosts, and detail the clinical features associated with concomitant nasal colonization.

Methods. Swabs of the bilateral anterior nares were obtained from patients diagnosed with MRSA bacteremia. A single colony culture from the blood and an average of 6 colonies from the nares were evaluated for MRSA growth. For the nares cultures, we typed multiple isolates for staphylococcal protein A (spa) and derived the clonal complexes. Demographic and clinical data were obtained retrospectively from the electronic medical record system and analysed using univariate and multivariable regression models.

Results. Over an 11-month period, 68 patients were diagnosed with MRSA bloodstream infection, 53 were swabbed, and 37 (70\%) were colonized with MRSA in the anterior nares. We performed molecular typing on 210 nasal colonies. Spa types and clonal complexes found in the blood were also detected in the nare in $95 \%$ of the cases. We also found that $11 \%$ of patients carried more than one clone of MRSA in the nares. Male sex and history of prior hospitalization within the past 90 days increased odds for MRSA colonization.

Conclusion. The molecular epidemiological landscape of colonization in the setting of invasive disease is diverse and defining the interplay between colonization and invasive disease is critical to combating invasive MRSA disease.

\section{Background}

Staphylococcus aureus ( $S$. aureus) is one of the most common causes of healthcare-associated infections (HAl), associated with high mortality rates in invasive disease $[1,2] . S$. aureus is also a common commensal pathogen and approximately $20-36 \%$ of the human population may be intermittently or persistently colonized, often asymptomatically [3-5]. Although $S$. aureus can colonize all skin surfaces, the most frequently assessed site of colonization is the anterior nares [6]. Nasal carriage plays a key role in progression onward to invasive disease [7], and persistent carriers have higher relative risk to develop bacteremia compared to non-carriers [8].

Within-host diversity of nasal carriage with $S$. aureus has been estimated to be $6.6 \%$ [9], and people commonly become infected with one of their colonizing isolates [10]. In a multicenter study, $82 \%$ of patients with MRSA bacteremia had identical clones in the anterior nares, and $86 \%$ of patients with MRSA colonization in anterior nares had clonally identical isolates later obtained from blood [10]. Colonization 
is also key to subtle transmission events and has been a central feature of outbreaks $[11,12]$. In the United States, most $S$. aureus infections are caused by two dominant clonal complexes, the clonal complex (CC) 5 and the CC8[2]. The CC 5 has historically encompassed hospital-associated genotypes, and the CC8 the community genotypes. As our work [13] and others [14] [15] have found these differences and their correlations are merging, we tested the associations between infection with dominant MRSA genotypes and the salient clinical features for the study population.

Given the importance of $S$. aureus colonization in invasive disease, we investigated the molecular epidemiological landscape of patients with MRSA bloodstream infections (BSI) with concomitant nasal colonization. Our aim was to collect a contemporary set of colonizing isolates from those with MRSA BSI, evaluate the frequency of diversity in the colonizing isolates, and define clinical features associated with the presence of colonization. We hypothesized that for patients with active MRSA BSI, there would be clinical differences between those concomitantly colonized and those who were not, as the presence of colonization itself is an important marker of disease status. We thus examined the clinical features and outcomes of patients with active MRSA BSI both with and without concomitant nasal colonization. Further definition of the link between colonization and infection will enable future avenues for mitigation of this infection.

\section{Results}

\section{Molecular diversity of nasal colonization in those with MRSA BSI}

Over an 11-month period (July 2018 to May 2019), 68 unique patients had MRSA BSI. Sequencing of single colonies from the blood cultures revealed that 30 (44\%) were caused by CC8, 28 (41\%) by CC5 and $10(15 \%)$ by other clonal complexes (Figure 1). From the 68 patients with MRSA BSI, 53 were successfully swabbed in the nares and screened for MRSA colonization. Fifteen patients were excluded due to discharge or death at the time of identification. From the 53 individuals who underwent nasal swabbing, $37(70 \%)$ were colonized with MRSA. The average number of colonies picked from the nasal growth plates was 6 (range 2 -12), resulting in a total of 210 which underwent spa typing (Supplementary Table 1). It was found that $95 \%$ of patients had at least one matching spa type between the blood isolate and nasal isolate. Four patients $(11 \%)$ carried multiple spa types in the anterior nares, resulting in a total 43 distinct nasal clones. Among the individuals who had swabs positive for MRSA, 23 of the nasal spa types corresponded to the CC5 and 15 corresponded to CC8, and 5 with other CCs (Figure 1). Within the CC5 samples, t002 represented the most common spa type, present in 15 of the 23 samples (65\%), while the t008 presented as the most common spa type amongst the CC8 samples, in 9 of the $15(60 \%)$ (Supplementary Table 1).

\section{Epidemiological and clinical characteristics associated with bacteremia and colonization}


The 68 patients with MRSA BSI were predominantly male $60 \%(n=41)$, between the age of 55 and $69(n=$ $28 ; 41 \%)$. The patients were $34 \%(n=23)$ non-Hispanic white, $34 \%(n=23)$ non-Hispanic black, $28 \%(n=$ 19) Hispanic, and $4 \%(n=3)$ belonged to other races or ethnicity groups (Table 1$)$. Patients were more frequently admitted from home $(n=46 ; 68 \%)$, but more than half of the patients had a history of previous hospitalization within the prior 90 days $(n=42 ; 62 \%)$. Community onset (CO) infections, or infections which present within the first 3 days of hospitalization, were more common compared to those associated with hospital onset $(\mathrm{HO})(n=40 ; 59 \%$ vs $n=28 ; 41 \%)$, however $75 \%(n=51)$ of patients had an invasive device in place at the time of infection. A prior personal history of colonization, as defined as any prior positive culture for MRSA from any site, or a documented history of prior MRSA infection or colonization, was present $28 \%$ of the time. The sources of infection were predominantly deemed to be unknown/other (32\%), vascular access (22\%), and skin and soft tissue $(21 \%)$, followed by peripheral IV (10\%), and pneumonia (7\%) (Table 1). 
Table 1

Demographics and Clinical Characteristics of Patients with MRSA BSI

\begin{tabular}{|c|c|}
\hline Factor, n (\%) & $(n=68)$ \\
\hline \multicolumn{2}{|l|}{ Sex } \\
\hline Male & $41(60)$ \\
\hline Female & $27(40)$ \\
\hline \multicolumn{2}{|l|}{ Race/Ethnicity } \\
\hline Non-Hispanic White & $23(34)$ \\
\hline Non-Hispanic Black & $23(34)$ \\
\hline Hispanic/Latinos & $19(28)$ \\
\hline Asian & $2(3)$ \\
\hline Other/Not Reported & $1(1)$ \\
\hline \multicolumn{2}{|l|}{ Age at Time of Infection } \\
\hline 18-54 Years & $18(26)$ \\
\hline 55-69 Years & $28(41)$ \\
\hline$\geq 70$ Years & $22(32)$ \\
\hline History of IV Drug Use & $4(6)$ \\
\hline HIV & $7(10)$ \\
\hline \multicolumn{2}{|l|}{ Admission Source } \\
\hline${ }^{\mathrm{a}}$ Home & $46(68)$ \\
\hline NH/Rehab/LTACH & $15(22)$ \\
\hline Outside Hospital & $7(10)$ \\
\hline Prior Hospital Admission (90 Days) & $42(62)$ \\
\hline \multicolumn{2}{|l|}{ Length of Hospital Stay Prior to BSI } \\
\hline CO-MRSA & $40(59)$ \\
\hline HO-MRSA & $28(41)$ \\
\hline \multicolumn{2}{|l|}{ Clonal Complex } \\
\hline CC5 & $28(41)$ \\
\hline CC8 & $30(44)$ \\
\hline
\end{tabular}




\begin{tabular}{|c|c|}
\hline Factor, $\mathrm{n}(\%)$ & $(n=68)$ \\
\hline Other & $10(15)$ \\
\hline \multicolumn{2}{|l|}{ Frequent Healthcare Interaction } \\
\hline Hemodialysis & $14(21)$ \\
\hline bInfusion Center & $14(21)$ \\
\hline None & $40(59)$ \\
\hline${ }^{c}$ Presence of Invasive Device & $51(75)$ \\
\hline${ }^{d}$ Invasive Procedures (missing $=1$ ) & $25(37)$ \\
\hline${ }^{e}$ Wound Present & $35(51)$ \\
\hline \multicolumn{2}{|l|}{${ }^{f}$ Comorbidities } \\
\hline Myocardial Infarction & $11(16)$ \\
\hline Congestive Heart Failure & $18(26)$ \\
\hline Peripheral Vascular Disease & $11(16)$ \\
\hline Cerebrovascular Disease & $11(16)$ \\
\hline Dementia & $8(12)$ \\
\hline Chronic Pulmonary Disease & $15(22)$ \\
\hline Connective Tissue Disease & $3(4)$ \\
\hline Peptic Ulcer Disease & $1(1)$ \\
\hline Mild Liver Disease & $4(6)$ \\
\hline Diabetes (no complications) & $18(26)$ \\
\hline Diabetes with Organ Damage & $11(16)$ \\
\hline Hemi or Paraplegia & $7(10)$ \\
\hline Moderate/Severe Renal Disease & $14(21)$ \\
\hline Solid Tumor & $2(3)$ \\
\hline Leukemia & $1(1)$ \\
\hline Lymphoma/Multiple Myeloma & $10(15)$ \\
\hline Moderate/Severe Liver Disease & $5(7)$ \\
\hline Metastatic Solid Tumor & $11(16)$ \\
\hline
\end{tabular}




\begin{tabular}{|ll|}
\hline Factor, $\mathbf{n}$ (\%) & $(\boldsymbol{n}=68)$ \\
\hline Charlson Comorbidity Index (CCI) & $13(19)$ \\
\hline $0-3$ & $20(29)$ \\
\hline $4-5$ & $22(32)$ \\
\hline $6-8$ & $13(19)$ \\
\hline$>8$ & $8(12)$ \\
\hline${ }^{g}$ History of Transplant & $19(28)$ \\
\hline hHistory of MRSA Colonization & \\
\hline Presumed Source of MRSA BSI & $7(10)$ \\
\hline Peripheral IV & $14(21)$ \\
\hline Skin \& Soft Tissue Infection & $5(7)$ \\
\hline Pneumonia & $1(1)$ \\
\hline Diabetic Foot Infection & $15(22)$ \\
\hline Vascular Access & $4(6)$ \\
\hline Sacral Wound & $22(32)$ \\
\hline iOther/Unknown Source & \\
\hline $\begin{array}{l}\text { Abbreviations: IV, intravenous; NH, nursing home; rehab, rehabilitation facility; LTACH, long-term acute } \\
\text { care hospital; CO, community-onset; HO-hospital-onset; MRSA, methicillin-resistant Staphylococcus }\end{array}$ & \\
\hline aureus; BSI, bloodstream infection; HIV, human immunodeficiency virus & \\
\hline & \\
\hline
\end{tabular}

a "Admission from home": included nonmedical residences such as home, group homes, assisted living facilities, and homeless shelters.

b "Infusion center": outpatient centers for chemotherapy, intravenous fluids, intravenous immunomodulators, and blood products.

c "Presence of invasive device": included pacemaker, implantable cardioverter defibrillator (ICD), left ventricular assist device (LVAD), vascular access (excluding peripheral intravenous catheters), orthopedic hardware, nephrostomy, suprapubic catheter, ileal conduit, foley catheter, arteriovenous graft placement (AVG), percutaneous endoscopic gastrostomy (PEG) tube, or ostomy.

$d$ "Invasive procedures": included any invasive procedures or surgery occurring within 1 month before first positive blood culture for MRSA, excluding electroencephalogram (EEG), electrocardiogram (EKG), and transthoracic echocardiogram (TTE).

e "Wound present": presence of a chronic skin wound overlying the sacrum, limb, abdomen, or other body part. 
f "Comorbidities": as defined by the Charleston Comorbidity Index (CCl); refer to standard definitions for $\mathrm{CCl}$

g "History of transplant": included solid organ and bone marrow transplant.

h "History of MRSA colonization": any positive culture from urine, sputum, tissue, or nares with MRSA prior to the positive MRSA blood culture or a documented history of prior MRSA infection or colonization.

i "Other/Unknown source": MRSA infection from urinary source, osteomyelitis, surgical site infection, spinal infection, septic arthritis or cardiac device infection, or an unknown/not reported source.

Multivariable analysis found that those with concomitant MRSA colonization were more likely to be male, comprising $73 \%(n=27)$ of the colonized patients compared to $44 \%(n=7)$ in the non-colonized group (OR, 5.06; 95\% Cl, $1.64-22.01 ; p=0.03$ ) (Table 2). Patients were more likely to have a prior hospital admission within 90 days $(n=26 ; 70 \%)$ compared to the non-colonized $(n=6 ; 38 \%)(O R, 3.96 ; 95 \% \mathrm{Cl}$, $1.01-15.56 ; p=0.05$ ). Patients with a history of renal disease including need for hemodialysis were more likely to be colonized $(\mathrm{OR}, 15.52 ; 95 \% \mathrm{Cl}, 1.13-213.37 ; p=0.04)$ while dementia was negatively associated with colonization ( $\mathrm{OR}, 0.10 ; 95 \% \mathrm{Cl}, 0.01-1.02 ; p=0.05)$ (Supplementary Table 2). There were no significant differences in outcomes between the two groups (Supplementary Table 3). 
Table 2

Demographics and Clinical Characteristics of Patients with MRSA BSI with and without nasal colonization

\begin{tabular}{|c|c|c|c|c|c|c|}
\hline Factor & $\begin{array}{l}\text { Colonized } \\
\begin{array}{l}\mathrm{N}=37 \\
(\%)\end{array}\end{array}$ & $\begin{array}{l}\text { Not } \\
\text { Colonized } \\
\mathrm{N}=16(\%)\end{array}$ & \multicolumn{2}{|c|}{$\begin{array}{l}\text { Univariate } \\
\text { Analysis } \\
\text { OR }(95 \% \mathrm{Cl}) p \text { value }\end{array}$} & \multicolumn{2}{|c|}{$\begin{array}{l}\text { Multivariable } \\
\text { Analysis } \\
\text { OR }(95 \% \mathrm{Cl}) p \text { value }\end{array}$} \\
\hline \multicolumn{7}{|l|}{ Sex } \\
\hline Male & $27(73)$ & $7(44)$ & $\begin{array}{l}3.47(1.02- \\
11.82)\end{array}$ & 0.05 & $\begin{array}{l}5.06(1.64- \\
22.01)\end{array}$ & 0.03 \\
\hline Female & $10(27)$ & $9(56)$ & Reference & & Reference & \\
\hline \multicolumn{7}{|l|}{ Race/Ethnicity } \\
\hline Non-Hispanic White & $13(35)$ & $7(44)$ & Reference & & & \\
\hline Non-Hispanic Black & $11(30)$ & $4(25)$ & $\begin{array}{l}1.48(0.34- \\
6.43)\end{array}$ & 0.60 & & \\
\hline Hispanic/Latino & $11(30)$ & $5(31)$ & $\begin{array}{l}1.19(0.29- \\
4.81)\end{array}$ & 0.81 & & \\
\hline Asian & $1(3)$ & $0(0)$ & -- & - & & \\
\hline Other/Not Reported & $1(3)$ & $0(0)$ & - & - & & \\
\hline \multicolumn{7}{|c|}{ Age at Time of Infection } \\
\hline 18-54 Years & $12(32)$ & $2(13)$ & Reference & & & \\
\hline 55-69 Years & $16(43)$ & $6(38)$ & $\begin{array}{l}0.44(0.08- \\
2.60)\end{array}$ & 0.37 & & \\
\hline$\geq 70$ Years & $9(24)$ & $8(50)$ & $\begin{array}{l}0.19(0.03- \\
1.11)\end{array}$ & 0.06 & & \\
\hline \multicolumn{7}{|l|}{ History of IV Drug Use } \\
\hline Yes & $2(5)$ & $1(6)$ & $\begin{array}{l}0.86(0.07- \\
10.19)\end{array}$ & 0.90 & & \\
\hline No & $35(95)$ & $15(94)$ & Reference & & & \\
\hline
\end{tabular}

See Table 1 for definitions.

Bold = significant at $\leq 0.05$

Abbreviations: IV, intravenous; $\mathrm{NH}$, nursing home; rehab, rehabilitation facility; LTACH, long-term acute care hospital; CO, community-onset; HO-hospital-onset; MRSA, methicillin-resistant Staphylococcus aureus; BSI, bloodstream infection; HIV, human immunodeficiency virus 


\begin{tabular}{|c|c|c|c|c|c|}
\hline \multirow{2}{*}{$\begin{array}{l}\text { Factor } \\
\\
\text { Yes }\end{array}$} & \multirow{2}{*}{$\begin{array}{l}\text { Colonized } \\
\begin{array}{l}\mathrm{N}=37 \\
(\%)\end{array} \\
6(16)\end{array}$} & \multirow{2}{*}{$\begin{array}{l}\text { Not } \\
\text { Colonized } \\
\mathrm{N}=16(\%) \\
0(0)\end{array}$} & \multicolumn{2}{|c|}{$\begin{array}{l}\text { Univariate } \\
\text { Analysis } \\
\text { OR }(95 \% \mathrm{Cl}) p \text { value }\end{array}$} & $\begin{array}{l}\text { Multivariable } \\
\text { Analysis } \\
\text { OR }(95 \% \mathrm{Cl}) p \text { value }\end{array}$ \\
\hline & & & - & - & \\
\hline No & $31(84)$ & $16(100)$ & -- & - & \\
\hline \multicolumn{6}{|l|}{ Admission Source } \\
\hline Home & $22(59)$ & $13(81)$ & Reference & & \\
\hline NH/Rehab/LTACH & $10(27)$ & $2(13)$ & $\begin{array}{l}2.96(0.56- \\
15.63)\end{array}$ & 0.20 & \\
\hline Other Hospital & $5(14)$ & $1(6)$ & $\begin{array}{l}2.96(0.31- \\
28.14)\end{array}$ & 0.35 & \\
\hline \multicolumn{6}{|c|}{$\begin{array}{l}\text { Prior Hospital Admission } \\
\text { (90 Days) }\end{array}$} \\
\hline Yes & $26(70)$ & $6(38)$ & $\begin{array}{l}3.94(1.15- \\
13.52)^{-}\end{array}$ & 0.03 & $\begin{array}{ll}3.96(1.01- & 0.05 \\
15.56) & \end{array}$ \\
\hline No & $11(30)$ & $10(63)$ & Reference & & Reference \\
\hline \multicolumn{6}{|c|}{$\begin{array}{l}\text { Length of Hospital Stay } \\
\text { Prior to BSI }\end{array}$} \\
\hline CO-MRSA & $20(54)$ & $10(63)$ & Reference & & \\
\hline HO-MRSA & $17(46)$ & $6(38)$ & $\begin{array}{l}1.42(0.43- \\
4.71)\end{array}$ & 0.57 & \\
\hline \multicolumn{6}{|l|}{ Clonal Complex } \\
\hline CC8 & $13(35)$ & $9(56)$ & Reference & & \\
\hline CC5 & $19(51)$ & $5(31)$ & $\begin{array}{l}2.63(0.72- \\
9.66)\end{array}$ & 0.15 & \\
\hline Other & $5(14)$ & $2(13)$ & $\begin{array}{l}1.73(0.27- \\
10.97)\end{array}$ & 0.56 & \\
\hline $\begin{array}{l}\text { Frequent Healthcare } \\
\text { Interaction }\end{array}$ & & & & & \\
\hline
\end{tabular}

See Table 1 for definitions.

Bold $=$ significant at $\leq 0.05$

Abbreviations: IV, intravenous; $\mathrm{NH}$, nursing home; rehab, rehabilitation facility; LTACH, long-term acute care hospital; CO, community-onset; HO-hospital-onset; MRSA, methicillin-resistant Staphylococcus aureus; BSI, bloodstream infection; HIV, human immunodeficiency virus 


\begin{tabular}{|c|c|c|c|c|c|c|}
\hline \multirow{2}{*}{$\begin{array}{l}\text { Factor } \\
\text { Hemodialysis }\end{array}$} & \multirow{2}{*}{$\begin{array}{l}\text { Colonized } \\
\begin{array}{l}\mathrm{N}=37 \\
(\%)\end{array} \\
12(32)\end{array}$} & \multirow{2}{*}{$\begin{array}{l}\begin{array}{l}\text { Not } \\
\text { Colonized }\end{array} \\
N=16(\%) \\
1(6)\end{array}$} & \multicolumn{2}{|c|}{$\begin{array}{l}\text { Univariate } \\
\text { Analysis } \\
\text { OR }(95 \% \mathrm{Cl}) p \text { value }\end{array}$} & \multicolumn{2}{|c|}{$\begin{array}{l}\text { Multivariable } \\
\text { Analysis } \\
\text { OR }(95 \% \mathrm{Cl}) p \text { value }\end{array}$} \\
\hline & & & $\begin{array}{l}8.00(0.92- \\
69.84)\end{array}$ & 0.06 & & \\
\hline Infusion Center & $7(19)$ & $3(19)$ & $\begin{array}{l}1.56(0.33- \\
7.24)\end{array}$ & 0.57 & & \\
\hline None & $18(49)$ & $12(75)$ & Reference & & & \\
\hline \multicolumn{7}{|c|}{$\begin{array}{l}\text { Presence of Invasive } \\
\text { Device }\end{array}$} \\
\hline Yes & $30(81)$ & $10(63)$ & $\begin{array}{l}2.57(0.70- \\
9.48)\end{array}$ & 0.16 & & \\
\hline No & $7(19)$ & $6(38)$ & Reference & & & \\
\hline \multicolumn{7}{|c|}{ Invasive Procedures } \\
\hline Yes & $13(35)$ & $4(25)$ & $\begin{array}{l}1.63(0.44- \\
6.07)\end{array}$ & 0.47 & & \\
\hline No & $24(65)$ & $12(75)$ & Reference & & & \\
\hline \multicolumn{7}{|l|}{ Wound Present } \\
\hline Yes & $21(57)$ & $6(38)$ & $\begin{array}{l}2.19(0.66- \\
7.29)\end{array}$ & 0.20 & & \\
\hline No & $16(43)$ & $10(63)$ & Reference & & & \\
\hline \multicolumn{7}{|c|}{$\begin{array}{l}\text { Charlson Comorbidity } \\
\text { Index (CCI) }\end{array}$} \\
\hline $0-3$ & $4(11)$ & $5(31)$ & Reference & & Reference & \\
\hline $4-5$ & $12(32)$ & $4(25)$ & $\begin{array}{l}3.75(0.66- \\
21.25)\end{array}$ & 0.14 & $\begin{array}{l}7.44(0.95- \\
58.45)\end{array}$ & 0.06 \\
\hline $6-8$ & $15(41)$ & $4(25)$ & $\begin{array}{l}4.69(0.84- \\
26.08)\end{array}$ & 0.08 & $\begin{array}{l}7.84(1.03- \\
59.84)\end{array}$ & 0.05 \\
\hline$>8$ & $6(16)$ & $3(19)$ & $\begin{array}{l}2.50(0.37- \\
16.89)\end{array}$ & 0.35 & $\begin{array}{l}3.68(0.44- \\
30.88)\end{array}$ & 0.23 \\
\hline
\end{tabular}

See Table 1 for definitions.

Bold = significant at $\leq 0.05$

Abbreviations: IV, intravenous; $\mathrm{NH}$, nursing home; rehab, rehabilitation facility; LTACH, long-term acute care hospital; CO, community-onset; HO-hospital-onset; MRSA, methicillin-resistant Staphylococcus aureus; BSI, bloodstream infection; HIV, human immunodeficiency virus 


\begin{tabular}{|c|c|c|c|c|c|}
\hline Factor & $\begin{array}{l}\text { Colonized } \\
N=37 \\
(\%)\end{array}$ & $\begin{array}{l}\begin{array}{l}\text { Not } \\
\text { Colonized }\end{array} \\
N=16(\%)\end{array}$ & \multicolumn{2}{|c|}{$\begin{array}{l}\text { Univariate } \\
\text { Analysis } \\
\text { OR }(95 \% \mathrm{Cl}) p \text { value }\end{array}$} & \multirow[t]{2}{*}{$\begin{array}{l}\text { Multivariable } \\
\text { Analysis } \\
\text { OR }(95 \% \mathrm{Cl}) p \text { value }\end{array}$} \\
\hline \multicolumn{5}{|l|}{ History of Transplant } & \\
\hline Yes & $6(16)$ & $1(6)$ & $\begin{array}{l}2.90(0.32- \\
26.33)\end{array}$ & 0.34 & \\
\hline No & $31(84)$ & $15(94)$ & Reference & & \\
\hline \multicolumn{6}{|l|}{$\begin{array}{l}\text { History of MRSA } \\
\text { Colonization }\end{array}$} \\
\hline Yes & $14(38)$ & $2(13)$ & $\begin{array}{l}4.26(0.84- \\
21.60)\end{array}$ & 0.08 & \\
\hline No & $23(62)$ & $14(88)$ & Reference & & \\
\hline \multicolumn{6}{|c|}{$\begin{array}{l}\text { Presumed Source of MRSA } \\
\text { BSI }\end{array}$} \\
\hline Peripheral IV & $5(14)$ & $2(13)$ & $\begin{array}{l}1.09(0.19- \\
6.33)\end{array}$ & 0.92 & \\
\hline $\begin{array}{l}\text { Skin \& Soft Tissue } \\
\text { Infection }\end{array}$ & $7(19)$ & $4(25)$ & $\begin{array}{l}0.70(0.17- \\
2.84)\end{array}$ & 0.62 & \\
\hline Pneumonia & $3(8)$ & $1(6)$ & $\begin{array}{l}1.32(0.13- \\
13.78)\end{array}$ & 0.81 & \\
\hline Diabetic Foot Infection & $1(3)$ & $0(0)$ & - & -- & \\
\hline Vascular Access & $11(30)$ & $3(19)$ & $\begin{array}{l}1.83(0.43- \\
7.74)\end{array}$ & 0.41 & \\
\hline Sacral Wound & $2(5)$ & $2(13)$ & $\begin{array}{l}0.40(0.05- \\
3.13)\end{array}$ & 0.38 & \\
\hline Other/Unknown & $8(22)$ & $4(25)$ & $\begin{array}{l}0.83(0.21- \\
3.28)\end{array}$ & 0.79 & \\
\hline \multicolumn{6}{|c|}{ See Table 1 for definitions. } \\
\hline \multicolumn{6}{|c|}{ Bold = significant at $\leq 0.05$} \\
\hline $\begin{array}{l}\text { Abbreviations: IV, intrav } \\
\text { care hospital; CO, comn } \\
\text { aureus; BSI, bloodstrea }\end{array}$ & $\begin{array}{l}\mathrm{NH} \text {, nursing } \\
\text { onset; HO-he } \\
\text { ction; HIV, hu }\end{array}$ & $\begin{array}{l}\text { home; rehab } \\
\text { spital-onset; } \\
\text { nan immunc }\end{array}$ & $\begin{array}{l}\text { ehabilitation } \\
\text { RSA, methic } \\
\text { eficiency virt }\end{array}$ & 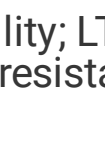 & $\begin{array}{l}\mathrm{CH} \text {, long-term acute } \\
\text { t Staphylococcus }\end{array}$ \\
\hline
\end{tabular}

When comparing clinical characteristics associated with two predominant clonal complexes (CC5 and CC8) in patients bacteremic during the entire study period, admission from a rehabilitation facility was significantly more common in those infected with CC5 $(n=11 ; 39 \%)$ compared to the CC8 $(n=4 ; 13 \%)$ 
(OR, 3.78; Cl $1.02-14.06 ; p=0.05$ )(Table 3). Furthermore, patients in the CC5 group had significantly higher rates of hospitalizations within the past 90 days $(n=22 ; 79 \%)$ compared to those with CC8 $(n=15$; $50 \%)(\mathrm{OR}, 3.67 ; \mathrm{Cl} 1.16-11.60 ; p=0.03)$. In the multivariable analysis, the presence of an invasive device was $93 \%(n=26)$ in the CC5 group and 60\% $(n=18)$ in the CC8 group (OR, 9.75; $\mathrm{Cl} 1.87-50.85 ; p=$ 0.007 ). Although not approaching significance, the primary source of BSI due to CC8 vs. CC5 was $13 \%$ vs $5 \%$ in skin derived infections (either due to peripheral IV or skin and soft tissue infection). While the 30day mortality rate for all bacteremic patients was $26 \%$, there were no differences in mortality and outcomes between those infected with either CC8 or CC5. 
Table 3

Demographics and Clinical Characteristics of Patients with MRSA BSI due to CC5 vs. CC8

\begin{tabular}{|c|c|c|c|c|c|c|}
\hline \multirow[t]{3}{*}{ Factor } & CC5 & CC8 & Univariate & & Multivariable & \\
\hline & \multirow{2}{*}{$\begin{array}{l}\mathrm{N}=28 \\
(\%)\end{array}$} & \multirow{2}{*}{$\begin{array}{l}\mathrm{N}=30 \\
(\%)\end{array}$} & \multicolumn{2}{|l|}{ Analysis $p$ value } & \multicolumn{2}{|c|}{ Analysis $p$ value } \\
\hline & & & \multicolumn{2}{|l|}{ OR (95\% Cl) } & \multicolumn{2}{|l|}{ OR (95\% Cl) } \\
\hline \multicolumn{7}{|l|}{ Sex } \\
\hline Male & $20(71)$ & $16(53)$ & $\begin{array}{l}2.19(0.74- \\
6.50)\end{array}$ & 0.16 & $\begin{array}{l}2.63(0.80- \\
8.61)\end{array}$ & 0.11 \\
\hline Female & $8(29)$ & $14(47)$ & Reference & & Reference & \\
\hline \multicolumn{7}{|l|}{ Race/Ethnicity } \\
\hline Non-Hispanic White & $10(36)$ & $11(37)$ & Reference & & & \\
\hline Non-Hispanic Black & $11(39)$ & $8(27)$ & $\begin{array}{l}1.51(0.43- \\
5.28)\end{array}$ & 0.52 & & \\
\hline Hispanic/Latino & $7(25)$ & $9(30)$ & $\begin{array}{l}0.86(0.23- \\
3.16)\end{array}$ & 0.82 & & \\
\hline Asian & $0(0)$ & $1(3)$ & - & - & & \\
\hline Other/Not Reported & $0(0)$ & $1(3)$ & - & - & & \\
\hline \multicolumn{7}{|c|}{ Age at Time of Infection } \\
\hline 18-54 Years & $7(25)$ & $8(27)$ & Reference & & & \\
\hline 55-69 Years & $9(32)$ & $15(50)$ & $\begin{array}{l}0.69(0.19- \\
2.54)\end{array}$ & 0.57 & & \\
\hline$\geq 70$ Years & $12(43)$ & $7(23)$ & $\begin{array}{l}1.96(0.49- \\
7.77)\end{array}$ & 0.34 & & \\
\hline \multicolumn{7}{|l|}{ History of IV Drug Use } \\
\hline Yes & $0(0)$ & $3(10)$ & - & - & & \\
\hline No & $\begin{array}{l}28 \\
(100)\end{array}$ & $27(90)$ & - & - & & \\
\hline
\end{tabular}

See Table 1 for definitions.

Bold = significant at $\leq 0.05$

Abbreviations: IV, intravenous; $\mathrm{NH}$, nursing home; rehab, rehabilitation facility; LTACH, long-term acute care hospital; CO, community-onset; HO-hospital-onset; MRSA, methicillin-resistant Staphylococcus aureus; BSI, bloodstream infection; HIV, human immunodeficiency virus 


\begin{tabular}{|c|c|c|c|c|c|}
\hline \multirow[t]{3}{*}{ Factor } & CC5 & CC8 & Univariate & & Multivariable \\
\hline & \multirow{2}{*}{$\underset{(\%)}{N}=28$} & \multirow{2}{*}{$\underset{(\%)}{\mathrm{N}=30}$} & \multicolumn{2}{|l|}{ Analysis $p$ value } & Analysis $p$ value \\
\hline & & & \multicolumn{2}{|l|}{ OR (95\% Cl) } & OR (95\% Cl) \\
\hline Yes & $3(11)$ & $3(10)$ & $\begin{array}{l}1.08(0.20- \\
5.86)\end{array}$ & 0.93 & \\
\hline No & $25(89)$ & $27(90)$ & Reference & & \\
\hline \multicolumn{6}{|l|}{ Admission Source } \\
\hline Home & $16(57)$ & $22(73)$ & Reference & & \\
\hline NH/Rehab/LTACH & $11(39)$ & $4(13)$ & $\begin{array}{l}3.78(1.02- \\
14.06)\end{array}$ & 0.05 & \\
\hline Other Hospital & $1(4)$ & $4(13)$ & $\begin{array}{l}0.34(0.04- \\
3.37)\end{array}$ & 0.36 & \\
\hline \multicolumn{6}{|c|}{$\begin{array}{l}\text { Prior Hospital Admission } \\
\text { (90 Days) }\end{array}$} \\
\hline Yes & $22(79)$ & $15(50)$ & $\begin{array}{l}3.67(1.16- \\
11.60)^{-}\end{array}$ & 0.03 & \\
\hline No & $6(21)$ & $15(50)$ & Reference & & \\
\hline \multicolumn{6}{|c|}{$\begin{array}{l}\text { Length of Hospital Stay } \\
\text { Prior to BSI }\end{array}$} \\
\hline CO-MRSA & $17(61)$ & $19(63)$ & Reference & & \\
\hline HO-MRSA & $11(39)$ & $11(37)$ & $\begin{array}{l}1.12(0.39- \\
3.23)\end{array}$ & 0.84 & \\
\hline \multicolumn{6}{|l|}{$\begin{array}{l}\text { Frequent Healthcare } \\
\text { Interaction }\end{array}$} \\
\hline Hemodialysis & $9(32)$ & $4(13)$ & $\begin{array}{l}3.00(0.77- \\
11.62)\end{array}$ & 0.11 & \\
\hline Infusion Center & $4(14)$ & $6(20)$ & $\begin{array}{l}0.89(0.21- \\
3.72)\end{array}$ & 0.87 & \\
\hline None & $15(54)$ & $20(67)$ & Reference & & \\
\hline
\end{tabular}

See Table 1 for definitions.

Bold = significant at $\leq 0.05$

Abbreviations: IV, intravenous; $\mathrm{NH}$, nursing home; rehab, rehabilitation facility; LTACH, long-term acute care hospital; CO, community-onset; HO-hospital-onset; MRSA, methicillin-resistant Staphylococcus aureus; BSI, bloodstream infection; HIV, human immunodeficiency virus 


\begin{tabular}{|c|c|c|c|c|c|c|}
\hline \multirow[t]{3}{*}{ Factor } & \multirow{3}{*}{$\begin{array}{l}\text { CC5 } \\
N=28 \\
(\%)\end{array}$} & \multirow{3}{*}{$\begin{array}{l}\text { CC8 } \\
\begin{array}{l}N=30 \\
(\%)\end{array}\end{array}$} & \multicolumn{2}{|l|}{ Univariate } & \multicolumn{2}{|c|}{ Multivariable } \\
\hline & & & \multicolumn{2}{|c|}{ Analysis $p$ value } & \multicolumn{2}{|c|}{ Analysis $p$ value } \\
\hline & & & OR (95\% Cl) & & OR $(95 \% \mathrm{Cl})$ & \\
\hline Yes & $26(93)$ & $18(60)$ & $\begin{array}{l}8.67(1.73- \\
43.49)\end{array}$ & 0.009 & $\begin{array}{l}9.75(1.87- \\
50.85)\end{array}$ & 0.007 \\
\hline No & $2(7)$ & $12(40)$ & Reference & & & \\
\hline \multicolumn{7}{|c|}{$\begin{array}{l}\text { Invasive Procedures } \\
\text { (missing = 1) }\end{array}$} \\
\hline Yes & $10(36)$ & $11(37)$ & $\begin{array}{l}0.91(0.31- \\
2.67)\end{array}$ & 0.86 & & \\
\hline No & $18(64)$ & $18(60)$ & Reference & & & \\
\hline \multicolumn{7}{|c|}{ Wound Present } \\
\hline Yes & $18(64)$ & $12(40)$ & $\begin{array}{l}2.70(0.93- \\
7.82)\end{array}$ & 0.07 & & \\
\hline No & $10(36)$ & $18(60)$ & Reference & & & \\
\hline \multicolumn{7}{|c|}{$\begin{array}{l}\text { Charlson Comorbidity Index } \\
\text { (CCI) }\end{array}$} \\
\hline $0-3$ & $5(18)$ & $7(23)$ & Reference & & & \\
\hline $4-5$ & $9(32)$ & $8(27)$ & $\begin{array}{l}1.58(0.35- \\
7.00)\end{array}$ & 0.55 & & \\
\hline $6-8$ & $11(39)$ & $8(27)$ & $\begin{array}{l}1.93(0.45- \\
8.33)\end{array}$ & 0.38 & & \\
\hline$>8$ & $3(11)$ & $7(23)$ & $\begin{array}{l}0.60(0.10- \\
3.54)\end{array}$ & 0.57 & & \\
\hline \multicolumn{7}{|c|}{ History of Transplant } \\
\hline Yes & $4(14)$ & $1(3)$ & $\begin{array}{l}4.83(0.51- \\
46.18)\end{array}$ & 0.17 & & \\
\hline No & $24(86)$ & $29(97)$ & Reference & & & \\
\hline $\begin{array}{l}\text { History } \\
\text { Coloniz }\end{array}$ & & & & & & \\
\hline
\end{tabular}

See Table 1 for definitions.

Bold = significant at $\leq 0.05$

Abbreviations: IV, intravenous; $\mathrm{NH}$, nursing home; rehab, rehabilitation facility; LTACH, long-term acute care hospital; CO, community-onset; HO-hospital-onset; MRSA, methicillin-resistant Staphylococcus aureus; BSI, bloodstream infection; HIV, human immunodeficiency virus 


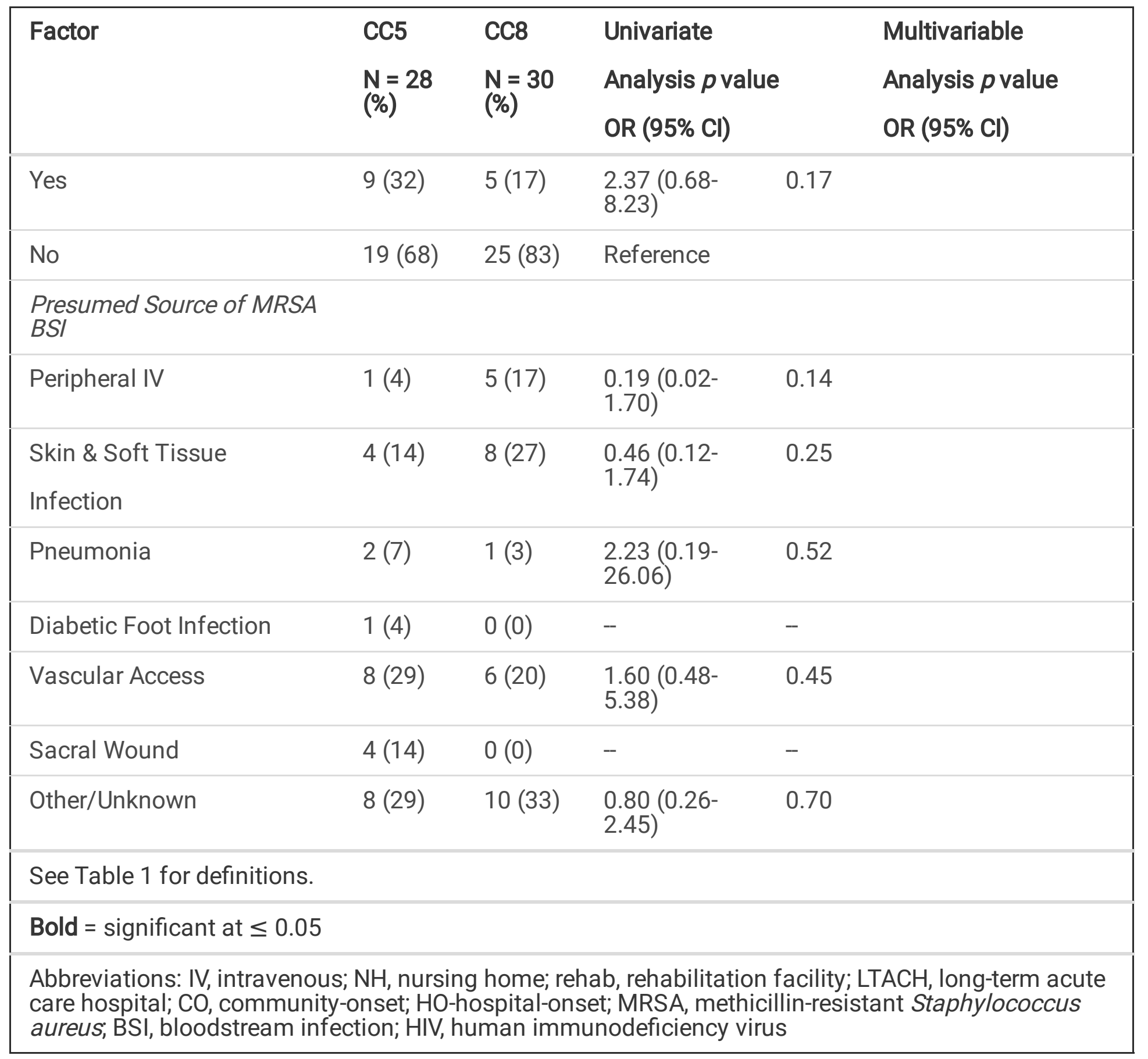

\section{Methods}

\section{Study setting, patient identification, and isolate selection}

The Mount Sinai Hospital (MSH) is a quaternary care facility. Under the approval of the MSH institutional review board (HS \# 18-00662; 13-00981), adults ( $\geq 18$ years old) with MRSA BSI were identified by the MSH Clinical Microbiology Laboratory as part of standard clinical care between July 2018 to May 2019. Identification and susceptibility of MRSA was performed by the Clinical Microbiology Laboratory using VITEK®2 (bioMerieux). Per hospital policy, all patients with MRSA BSI are placed on contact isolation with staff donning gown and gloves for interactions. Swabbers washed their hands prior to donning 
gown and gloves, and additionally wore surgical masks during sample collection. Bilateral anterior nares were sampled using a single, pre-labeled, sterile swab (Puritan ${ }^{\circledR}$ PurFlock®) Ultra Sterile Flocked Collection Devices) moisturized with sterile $0.9 \% \mathrm{NaCl}$ saline solution inserted up to $2.5 \mathrm{~cm}$ from the edge of the nares and rolled 5 times in each nostril. Swabs were plated directly on CHROMID® MRSA plates. After 24 hours of incubation at $37^{\circ} \mathrm{C}$, the plate was evaluated for positive MRSA growth. If positive for MRSA, the isolate lawns were collected and used to inoculate Tryptic Soy Broth (TSB) with $15 \%$ glycerol cryovials. If negative for growth, the plates were re-evaluated at 48 hours. When needed, Staphaurex ${ }^{\text {TM }}$ Latex Agglutination Test was used to confirm coagulase positivity. The cryovial contents were homogenized using a Vortex-Genie 2 and then stored in a $-80^{\circ} \mathrm{C}$ freezer.

For the molecular work up, the frozen stocks from the nasal swab stocks were plated on BD BBL ${ }^{\mathrm{TM}}$ Trypticase ${ }^{\text {TM }}$ Soy Agar (TSA II ${ }^{\text {TM }}$ ) with Sheep Blood and incubated at $37^{\circ} \mathrm{C}$. Selection of colonies was performed using a previously described replica plating technique [16] in which nasal swab stocks were diluted in $1 \mathrm{X}$ PBS and spread onto TSA II plates before incubation for 16 hours at $37^{\circ} \mathrm{C}$ to facilitate an even spread of distinct colonies. From these base plates we replica plated onto new TSA II Blood plate, BD BBL ${ }^{\mathrm{TM}}$ Prepared Plated Media: Mannitol Salt Agar, CHROMID ${ }^{\circledR}$ MRSA plates, and BD BBL ${ }^{\mathrm{T}}$ Oxacillin Screen Agar (Mueller Hinton Agar w/6 $\mathrm{gg} / \mathrm{mL}$ Oxacillin, 4\% $\mathrm{NaCl}$ ) Prepared Media plates, respectively. These replica plates were grown at $37^{\circ} \mathrm{C}$ for 24 hours before examination for phenotypic differences in colony morphology. The aim was to select approximately 6 (ideally morphologically diverse) colonies (Supplementary Table 1) for every swab sample, and bacterial stocks were prepared using TSB with 15\% glycerol. All colonies were analysed using a Bruker MALDI Biotyper® System to confirm the growth of $S$. aureus.

\section{Molecular Typing}

Single colonies from the clinical MRSA blood cultures underwent DNA extraction and PacBio whole genome sequencing as part of a surveillance program, as described previously [11]. From the resulting genomes a custom script (https://github.com/mjsull/spa_typing) was run to identify the spa type. The raw sequence data for the blood genomes have been deposited in the National Center for Biotechnology Information SRA database under Bioproject PRJNA470993.

DNA extraction of the nasal cultures was performed on a ThermoFisher KingFisher Flex 96 using Applied BioSystems MagMAX ${ }^{\mathrm{TM}}$ DNA Multi-Sample Ultra 2.0 Kits. Typing of the spa gene was performed by first using PCR to amplify the spa sequence through use of previously described methods [17] and Sanger sequencing performed by Psomagen Inc.

\section{Patient data collection and statistical analyses}

Under approval obtained from the Mount Sinai Hospital Institutional Review Board (HS\# 17-00825), demographic and clinical data were obtained retrospectively from the electronic medical record system, including admission data, presumed source of the BSI based on Infectious Diseases (ID) consults, comorbidities, and prior outpatient health care exposures. Chart abstraction and data entries were placed into the REDCap [18] system and verified by two different ID trained individuals. All patients diagnosed 
with MRSA BSI received an ID consultation at the time of diagnosis, as per standard practice at our institution. Surveillance definitions included hospital-onset MRSA (HO-MRSA), defined as positive cultures on or after the fourth day after hospital admission, and community-onset MRSA (CO-MRSA), defined as BSI presenting within the 72-hour hospital admission interval [19]. We also evaluated inhospital outcomes and death, especially those considered to be related to the MRSA BSI. We categorized all non-normally distributed continuous variables into discrete categorical groups. The univariate logistic regression model was performed initially, and variables with $p$ values $\leq .2$ were placed in the multivariable logistic regression model. All variables with $p \leq .05$ were considered statistically significant. All analyses were performed in SAS (version 9.4) [20].

\section{Discussion}

Colonization is an important feature of invasive MRSA disease. In our study we found that $70 \%$ of patients with MRSA BSI were colonized with MRSA in the anterior nares. In $95 \%$ of these cases, the MRSA clones present in the blood were also found in the nares, suggesting infection from an endogenous source.

As nasal colonization was associated with history of a recent hospitalization within the prior 90 days, presumably these individuals may become colonized with exposure to the healthcare setting. While baseline colonization is not assessed on admission at our hospital, nasal colonization with MRSA has been independently associated with healthcare exposure in prior studies [21]. The relationship between recent hospitalization and colonization was implicated through the higher rates of CC5 amongst those with prior healthcare exposures and higher overall rates of colonization with the CC5. This may signal differences in the avenues in which BSI due to CC5 and CC8 are acquired given the association of CC5 and the healthcare setting. Additionally, a higher proportion of male patients with MRSA BSI were found to be colonized when compared to women. This finding is consistent with prior studies and may be attributed to differences in the microbiome of the skin such as sweat, sebum, and hormone production along with differences in behavior and hand-hygiene practices [22, 23].

The overall genetic diversity found among the nasal clones was $11 \%$. However, within-patient nasal cultures exhibited a high degree of relatedness with one another, with most clustering into the same genetic clades. A prior study focused on nasal carriage clonality estimated a $6.6 \%$ diversity [9], while one pediatric study found $30 \%$ diversity over longitudinal time points [24], suggesting that carriage of discordant $S$. aureus clones in individuals with nasal colonization occur regularly and may lead to horizontal genetic exchange among clones. Nonetheless, there is a paucity of data regarding $S$. aureus diversity in nasal colonization. With evaluations limited to a small number of colonies, the actual diversity is likely greater [25].

There were few overall differences in the clinical characteristics of patients infected with either CC5 or CC8 MRSA, upholding prior findings that indicate that historical hospital and community associations of clones are indeed blurred in endemic regions. Skin and soft tissue and peripheral intravenous catheter 
(PIV) infections tended to correlate more frequently to the CC8 MRSA. While CC8 is associated with community skin derived infections [26], we have previously observed that CC8 was also associated with PIV infections [13] highlighting the importance of community clones causing hospital-onset infections. Colonization was associated with patients with prior history of renal disease, which is a well-established clinical association given the frequent interaction with healthcare facilities and likelihood of requiring vascular access [27].

There are some limitations in this study. The patient population at our institution is complex and might not represent the same population in smaller community hospitals. Regional and global differences in the prevalence of MRSA colonization and clonal types may limit generalizability. The retrospective chart review portion may be subject to errors in chart abstraction. Although we expect that the numerically dominant clones are most likely to be selected, particularly in the setting bacteremia, we are likely underrepresenting the full diversity [28]. While it is understood that people become infected with their colonizing isolate, we are unable to directly address directionality, as there may have been a single exposure that led to acquisition at both sites. Finally, we focused on MRSA-BSI due to the focus of our molecular surveillance program although we recognize that methicillin-susceptible $S$. aureus (MSSA) also causes significant disease [29].

\section{Conclusions}

Colonization is an essential component of invasive MRSA disease, and we found high rates of colonization, and colonization with more than one clone at a frequency of $11 \%$. This contemporary sample collection adds to the paucity of literature on the frequency and the diversity of colonization in the presence of active MRSA BSI. We found colonization to be associated with male sex and those with a recent hospitalization, highlighting potential high-risk groups. Combined molecular and clinical analyses will assist in defining the intra-host and inter-host dynamics of MRSA and enable the development of targeted approaches to curtail disease.

\section{Abbreviations}

HAls: Healthcare-associated infections

MRSA: Methicillin-resistant S. aureus

BSI: Bloodstream infections

spa: Staphylococcal protein A

S. aureus: Staphylococcus aureus

CC: Clonal complex

CO: Community onset 
MSH: Mount Sinai Hospital

TSB: Tryptic Soy Broth

TSA II ${ }^{\mathrm{TM}}$ : Trypticase ${ }^{\mathrm{TM}}$ Soy Agar

ID: Infectious diseases

HO-MRSA: Hospital-onset MRSA

CO-MRSA: Community-onset MRSA

MSSA: Methicillin-susceptible $S$. aureus

\section{Declarations}

\section{Availability of data and materials}

The raw sequence data for the blood genomes that support the findings of this study have been deposited in the National Center for Biotechnology Information SRA database under Bioproject PRJNA470993; other data used and/or analysed during the current study are available from the corresponding author on reasonable request.

\section{Ethics Statement}

The study protocols were reviewed and approved by the Icahn School of Medicine at Mount Sinai Institutional Review Board. Use and disclosure of protected health information (PHI) was approved under a waiver of authorization and a waiver of informed consent was obtained. Collection and processing of bacterial samples were covered with protocols HS \# 18-00662 and \# 13-00981, and the clinical data collection with the protocol HS \# 17-00825. This study was performed in accordance with the Helsinki Declaration of 1964 , and its later amendments.

\section{Consent for publication}

Not applicable.

\section{Competing interests}

The authors declare that they have no competing interests.

\section{Funding}

This research was supported in part by R01 Al119145 (H.v.B.). The funders had no role in the study design, data collection and interpretation, or the decision to submit the work for publication.

\section{Author Contributions}


ERS, AvdG, ACD, MC, DRA wrote the manuscript. ERS, AvdG, MC, DRA, MG, RS, HvB revised the manuscript. ERS, ACD, ABC, DN, LF, AM, RS, GP, DRA performed the clinical evaluations. AvdG, FS, JP, MG, MC, DRA, HvB performed analysis of clinical samples. ACD, DRA performed statistical analyses. AO, KC, HvB assisted with data collection. AvdG, IO, MJS, MLS, HvB performed isolate sequencing. All authors read and approved the final manuscript.

\section{Acknowledgements}

Not applicable

\section{Author information}

The authors Erika Reategui Schwarz and Adriana van de Guchte contributed equally to this work.

Affiliations

Division of Infectious Diseases, Department of Medicine, Icahn School of Medicine at Mount Sinai, New York City, New York.

Erika Reategui Schwarz, Amy C. Dupper, Ana Berbel Caban, Devika Nadkarni, Lindsey Fox, Alexandra Mills, Richard Silvera, MD, Gopi Patel, Melissa Gitman, Deena R. Altman.

Department of Genetics and Genomic Sciences, Icahn School of Medicine at Mount Sinai, New York City, New York.

Adriana van de Guchte, Ajay Obla, Irina Oussenko, Jose Polanco, Melissa L. Smith, Marilyn Chung PhD, Mitchell J. Sullivan, Harm van Bakel, Deena R. Altman.

Icahn Institute for Data Science and Genomic Technology, Icahn School of Medicine at Mount Sinai, New York City, New York.

Ajay Obla, Kieran I. Chacko, Melissa L. Smith, Harm van Bakel.

Department of Pathology, Icahn School of Medicine at Mount Sinai, New York City, New York. Melissa Gitman, Flora Samaroo.

\section{References}

1. Lowy FD. Staphylococcus aureus infections. N Engl J Med. 1998;339(8):520-32.

2. Chambers HF, Deleo FR. Waves of resistance: Staphylococcus aureus in the antibiotic era. Nat Rev Microbiol. 2009;7(9):629-41.

3. Wertheim HF, Melles DC, Vos MC, van Leeuwen W, van Belkum A, Verbrugh HA, et al. The role of nasal carriage in Staphylococcus aureus infections. Lancet Infect Dis. 2005;5(12):751-62. 
4. Muthukrishnan G, Lamers RP, Ellis A, Paramanandam V, Persaud AB, Tafur S, et al. Longitudinal genetic analyses of Staphylococcus aureus nasal carriage dynamics in a diverse population. BMC Infect Dis. 2013;13:221.

5. VandenBergh MF, Yzerman EP, van Belkum A, Boelens HA, Sijmons M, Verbrugh HA. Follow-up of Staphylococcus aureus nasal carriage after 8 years: redefining the persistent carrier state. $\mathrm{J}$ Clin Microbiol. 1999;37(10):3133-40.

6. Kluytmans J, van Belkum A, Verbrugh H. Nasal carriage of Staphylococcus aureus: epidemiology, underlying mechanisms, and associated risks. Clin Microbiol Rev. 1997;10(3):505-20.

7. Davis KA, Stewart JJ, Crouch HK, Florez CE, Hospenthal DR. Methicillin-resistant Staphylococcus aureus (MRSA) nares colonization at hospital admission and its effect on subsequent MRSA infection. Clin Infect Dis. 2004;39(6):776-82.

8. Wertheim HF, Vos MC, Ott A, van Belkum A, Voss A, Kluytmans JA, et al. Risk and outcome of nosocomial Staphylococcus aureus bacteraemia in nasal carriers versus non-carriers. Lancet. 2004;364(9435):703-5.

9. Cespedes C, Said-Salim B, Miller M, Lo SH, Kreiswirth BN, Gordon RJ, et al. The clonality of Staphylococcus aureus nasal carriage. J Infect Dis. 2005;191(3):444-52.

10. von Eiff C, Becker K, Machka K, Stammer H, Peters G. Nasal carriage as a source of Staphylococcus aureus bacteremia. Study Group. N Engl J Med. 2001;344(1):11-6.

11. Berbel Caban A, Pak TR, Obla A, Dupper AC, Chacko KI, Fox L, et al. PathoSPOT genomic epidemiology reveals under-the-radar nosocomial outbreaks. Genome Med. 2020;12(1):96.

12. Senn L, Clerc O, Zanetti G, Basset P, Prod'hom G, Gordon NC, et al. The Stealthy Superbug: the Role of Asymptomatic Enteric Carriage in Maintaining a Long-Term Hospital Outbreak of ST228 MethicillinResistant Staphylococcus aureus. mBio. 2016;7(1):e02039-15.

13. Dupper AC, Sullivan MJ, Chacko KI, Mishkin A, Ciferri B, Kumaresh A, et al. Blurred Molecular Epidemiological Lines Between the Two Dominant Methicillin-Resistant Staphylococcus aureus Clones. Open Forum Infect Dis. 2019;6(9):ofz302.

14. Seybold U, Kourbatova EV, Johnson JG, Halvosa SJ, Wang YF, King MD, et al. Emergence of community-associated methicillin-resistant Staphylococcus aureus USA300 genotype as a major cause of health care-associated blood stream infections. Clin Infect Dis. 2006;42(5):647-56.

15. Popovich KJ, Weinstein RA, Hota B. Are community-associated methicillin-resistant Staphylococcus aureus (MRSA) strains replacing traditional nosocomial MRSA strains? Clin Infect Dis. 2008;46(6):787-94.

16. Lederberg J, Lederberg EM. Replica plating and indirect selection of bacterial mutants. J Bacteriol. 1952;63(3):399-406.

17. Pardos de la Gandara M, Raygoza Garay JA, Mwangi M, Tobin JN, Tsang A, Khalida C, et al. Molecular Types of Methicillin-Resistant Staphylococcus aureus and Methicillin-Sensitive S. aureus Strains Causing Skin and Soft Tissue Infections and Nasal Colonization, Identified in Community Health Centers in New York City. J Clin Microbiol. 2015;53(8):2648-58. 
18. Harris PA, Taylor R, Thielke R, Payne J, Gonzalez N, Conde JG. Research electronic data capture (REDCap)-a metadata-driven methodology and workflow process for providing translational research informatics support. J Biomed Inform. 2009;42(2):377-81.

19. CDC. Multidrug-Resistant Organisms \& Clostrioides difficile Infection (MDRO/CDI) Module. 2018.

20. Base SAS 9.4 Procedures Guide: Statistical Procedures: SAS Institute Inc. SAS Institute; 2013.

21. Gorwitz RJ, Kruszon-Moran D, McAllister SK, McQuillan G, McDougal LK, Fosheim GE, et al. Changes in the prevalence of nasal colonization with Staphylococcus aureus in the United States, 2001-2004. J Infect Dis. 2008;197(9):1226-34.

22. Humphreys H, Fitzpatick F, Harvey BJ. Gender differences in rates of carriage and bloodstream infection caused by methicillin-resistant Staphylococcus aureus: are they real, do they matter and why? Clin Infect Dis. 2015;61(11):1708-14.

23. Grice EA, Segre JA. The skin microbiome. Nat Rev Microbiol. 2011;9(4):244-53.

24. Mongkolrattanothai K, Gray BM, Mankin P, Stanfill AB, Pearl RH, Wallace LJ, et al. Simultaneous carriage of multiple genotypes of Staphylococcus aureus in children. J Med Microbiol. 2011;60(Pt 3):317-22.

25. Paterson GK, Harrison EM, Murray GGR, Welch JJ, Warland JH, Holden MTG, et al. Capturing the cloud of diversity reveals complexity and heterogeneity of MRSA carriage, infection and transmission. Nat Commun. 2015;6:6560.

26. DeLeo FR, Otto M, Kreiswirth BN, Chambers HF. Community-associated meticillin-resistant Staphylococcus aureus. Lancet. 2010;375(9725):1557-68.

27. Zacharioudakis IM, Zervou FN, Ziakas PD, Mylonakis E. Meta-analysis of methicillin-resistant Staphylococcus aureus colonization and risk of infection in dialysis patients. J Am Soc Nephrol. 2014;25(9):2131-41.

28. Didelot X, Walker AS, Peto TE, Crook DW, Wilson DJ. Within-host evolution of bacterial pathogens. Nat Rev Microbiol. 2016;14(3):150-62.

29. Kourtis AP, Hatfield K, Baggs J, Mu Y, See I, Epson E, et al. Vital Signs: Epidemiology and Recent Trends in Methicillin-Resistant and in Methicillin-Susceptible Staphylococcus aureus Bloodstream Infections - United States. MMWR Morb Mortal Wkly Rep. 2019;68(9):214-9.

\section{Figures}




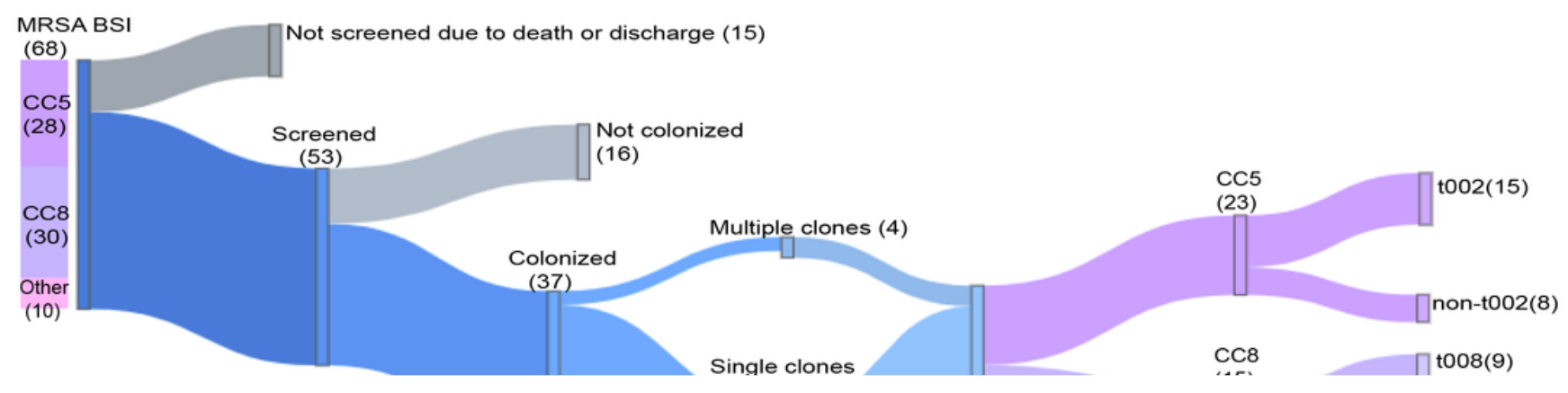

\section{Figure 1}

Sankey flow chart diagram of 68 patients with MRSA BSI detailing nasal colonization status with breakdown by spa and clonal complex (CC).

\section{Supplementary Files}

This is a list of supplementary files associated with this preprint. Click to download.

- SupplementaryTable1.xlsx

- SupplementaryTable2.docx

- SupplementaryTable3.docx 